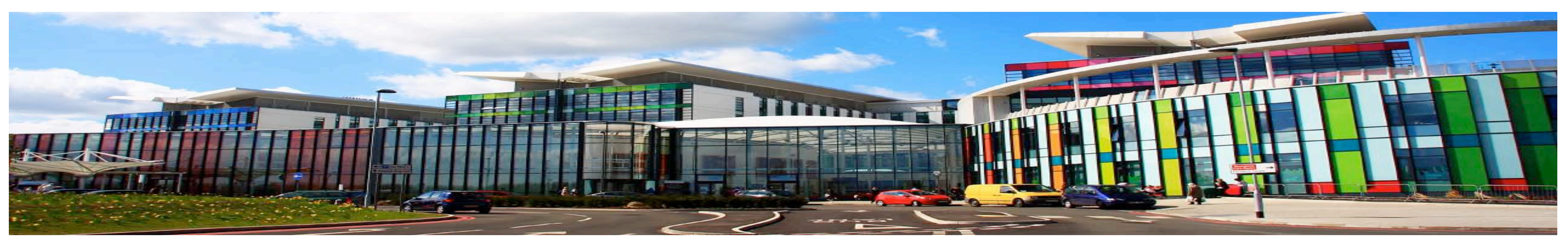

\title{
MEN -1 with Primary hyperparathyroidism in pregnancy: a report of two cases
}

\section{Dr Manjusha Rathi, Sherwood Forest Hospitals NHS Foundation Trust, Sutton-in-Ashfield, UK}

\section{Introduction:}

Primary hyperparathyroidism (PHPT) is the most common endocrinopathy in MEN1, reaching nearly $100 \%$ penetrance by age 50 years. MEN1, however, is rare in PHPT accounting for only 2-4\% of cases of PHPT. Incidence of MEN1 syndrome is 1/30,000 in general population and incidence of PHPT in reproductive age women is 8/100,000 population/year. However, coexistent of MEN1, PHPT and pregnancy is extremely rare and true incidence is unknown. PHPT in pregnancy remains high risk with significant morbidity and mortality despite improvements in medical and obstetric care.

\section{Method:}

Case 1: $26 \mathrm{yr}$ old Caucasian lady, known to have confirmed MEN1 gene mutation with previous distal pancreatectomy for neuroendocrine tumour; was closely monitored as these individuals have increased likelihood of developing pituitary tumours and primary hyperparathyroidism. During her second pregnancy, she was noted to have consistently high adjusted calcium was 2.71 (ref $2.2-2.6 \mathrm{mmol} / \mathrm{L}$ ) with raised parathyroid hormone and was diagnosed to have PHPT. She remained asymptomatic during pregnancy with serum adjusted calcium ranging between $2.67-2.72 \mathrm{mmol} / \mathrm{L}$ with satisfactory fetal growth. Therefore, she was managed conservatively with a eucalcaemic diet, good hydration and regular monitoring of maternal blood tests (serum urea, electrolytes, corrected calcium and phosphate) and serial fetal Growth scan. She delivered healthy boy at 39 weeks gestation. During post-partum period, neonate was closely monitored for development of hypocalcaemia and she underwent planned parathyroid surgery.

Case 2: a younger sister of case 1; with confirmed MEN-1 gene mutation was diagnosed to have PHPT age 14 years and had previously declined parathyroid surgery. At age 28 years, during her first pregnancy, her adjusted Calcium was noted to be high at $2.88 \mathrm{mmol} / \mathrm{L}$ in the first trimester. Risk associated with hypercalcaemia during pregnancy and need to have definite treatment in the form of parathyroid surgery were explained. However she declined surgery due to concerns related to surgical /anaesthetic risk including increased risk of fetal loss. At 20 weeks' gestation, fetal growth was below $5^{\text {th }}$ centile. Further scan performed 3 days later confirmed reduced blood flow in umbilical cord with abnormal umbilical artery Doppler PI.

In view of the findings of fetal growth restriction (FGR) and a raised umbilical artery Doppler PI, the need for Parathyroid surgery was revisited. However, she declined surgery. Despite close monitoring, adequate hydration, her calcium remained consistently above $2.8 \mathrm{mmol} / \mathrm{L}$. Subsequently, she developed pregnancy induced hypertension and at 24 weeks gestation, fetal scan confirmed intrauterine death.

\section{Discussion:}

PHPT in pregnancy is associated with increased maternal $(67 \%)$ and fetal $(80 \%)$ complications. Maternal complications include gestational hypertension, pre-eclampsia, nephrolithiasis, bone disease, pancreatitis, hyperemesis, mental status changes, and hypercalcaemic crisis. Fetal complications include intrauterine growth retardation, low birth weight, preterm delivery, intrauterine fetal demise, postpartum neonatal hypocalcaemia/ tetany, and permanent hypoparathyroidism.

In second case highlighted above, intrauterine growth retardation followed by death may well have been a secondary complication of PHPT due to its known association with FGR.

Current evidence suggests surgery should be performed preferably in the second trimester, if serum calcium levels are above $2.75 \mathrm{mmol} / \mathrm{L}$. However, women in early or late pregnancy should not be denied consideration of surgical treatment even when calcium levels are only moderately elevated, as the extent of maternal or fetal complications from PHPT cannot currently be predicted on serum calcium levels alone.

For patients who are unsuitable or decline parathyroid surgery, there is currently limited clinical data outlining the safety and efficacy of treatment with calcitonin and calcimimetics such as cinacalcet for PHPT in pregnancy.

\section{Conclusion:}

PHPT during pregnancy remains high risk and conservative intervention may be appropriate under certain circumstances; however, excision of a parathyroid adenoma remains the only definitive treatment to reduce the risk of adverse pregnancy outcome.

\section{References:}

1. Brandi ML, Gagel RF, Angeli A et al. Guidelines for diagnosis and therapy of MEN type 1 and type 2. J Clin Endocrinol Metab 2001; 86: 5658

2. Thakker RV, et al. Clinical practice guidelines for multiple endocrine neoplasia type 1 (MEN1). J Clin Endocrinol Metab 2012; 97: 2990-3011.

3. Diaz-Soto G, et al. Primary hyperparathyroidism in pregnancy. Endocrine 2013; 44: 591-597. 